\title{
A conformal approach for the analysis of the non-linear stability of pure radiation cosmologies
}

\author{
Christian Lübbe ${ }^{*, 1,2}$ and Juan Antonio Valiente Kroon ${ }^{\dagger, 2}$ \\ ${ }^{1}$ Department of Mathematics, University of Leicester, University Road, LE1 8RH, United Kingdom. \\ ${ }^{2}$ School of Mathematical Sciences, Queen Mary, University of London, Mile End Road, London E1 4NS, \\ United Kingdom.
}

August 29, 2018

\begin{abstract}
The conformal Einstein equations for a tracefree (radiation) perfect fluid are derived in terms of the Levi-Civita connection of a conformally rescaled metric. These equations are used to provide a non-linear stability result for de Sitter-like tracefree (radiation) perfect fluid Friedman-Lemaître-Robertson-Walker cosmological models. The solutions thus obtained exist globally towards the future and are future geodesically complete.
\end{abstract}

PACS: 04.20.Ex, 04.20.Ha, 98.80.Jk

\section{Introduction}

The conformal Einstein field equations have proven a powerful tool to analyse the stability and the global properties of vacuum, electro-vacuum and Yang-Mills-electro-vacuum spacetimes - see e.g. [9, 10, 11, 18, 19, 20, By contrast, to the best of our knowledge, there has been no attempt to make use of conformal methods to analyse similar issues in spacetimes whose matter content is given by a perfect fluid. In this article we make a first step in this direction. We discuss the stability and the global properties of a class of cosmological spacetimes having as a source a perfect fluid with tracefree energy-momentum tensor. The solutions we construct are non-linear perturbations of a Friedman-Lemaître-Robertson-Walker (FLRW) reference spacetime.

The present analysis is to be regarded as a first step in the development of conformal methods for the discussion of cosmological models whose matter content is described by a perfect fluid. Hence, we restrict our attention to the simplest case from the point of view of conformal methods: perturbations of a traceless prefect fluid cosmological model with compact spatial sections of positive constant curvature. Generalisation of our analysis to more general background solutions and equations of state will be discussed elsewhere.

The problem of the non-linear stability of FLRW cosmologies and the exponential decay of perturbations is considered in 23]. In that reference, a frame formulation of the Einstein-perfect fluid system [12] is used to obtain a suitable symmetric hyperbolic evolution system for which the Kreiss-Lorenz theory can be readily applied - see [17. The results obtained hold for a large class of equations of state, but not very stiff ones - like the pure radiation case discussed in the present article. More recently, the problem of the non-linear stability of the irrotational Euler-Einstein system for de Sitter-like spacetimes has been analysed in [24]. This analysis shows that FLRW background solutions with pressure $\tilde{p}$ and density $\tilde{\rho}$ related by a barotropic equations of state of the form $\tilde{p}=\gamma \tilde{\rho}$ with $1<\gamma<\frac{4}{3}$ are future asymptotically stable under small irrotational

\footnotetext{
${ }^{*}$ E-mail address:c.luebbe@qmul.ac.uk, cl242@le.ac.uk

${ }^{\dagger}$ E-mail address:j.a.valiente-kroon@qmul.ac.uk
} 
perturbations. An extension of this analysis to the case of fluids with non-zero vorticity has been given in [26].

It is notable that the case of a pure radiation perfect fluid cannot be covered by the analysis of [23, 24, 26]. By contrast, from the point of view of conformal methods, the pure radiation perfect fluid case turns out to be one of the simplest scenarios to be considered. Finally, it should be mentioned that conformal methods have been used to pose an initial value problem for the Einstein-Euler system at the Big Bang for a class of cosmological models with isotropic singularities - see [1]. The methods used in that work do not allow, however, to obtain global existence assertions towards the future.

Our main result can be stated as follows:

Theorem. Suppose one is given Cauchy initial data for the Einstein-Euler system with a de Sitter-like cosmological constant and equation of state for pure radiation. If the initial data is sufficiently close to data for a FLRW cosmological model with the same equation of state, value of the cosmological constant and spatial curvature $k=1$, then the development exists globally towards the future, is future geodesically complete and remains close to the FLRW solution.

A detailed and technically precise version of this result is given in Theorem 2

Remark 1. Similar future global existence and stability results can be obtained using the methods of this article for a FLRW background solution with pure radiation equation of state, de Sitter-like or vanishing cosmological constant, $\lambda$, and $k=0,-1$. These models expand indefinitely towards the future, and remarkably, their scale factor can be computed explicitly — see [14. In the cases with $\lambda=0$, minor technical modifications need to be introduced to account for a null conformal boundary. The stability of these models will be discussed elsewhere by means of different (conformal) methods.

Remark 2. The restriction of our analysis to the case of perfect fluids with traceless energymomentum tensor is technical: in this case the equation of conservation of energy momentum transforms homogeneously under conformal transformations. In the case of perfect fluids with an energy-momentum tensor with non-vanishing trace a regularisation of the rescaled equations of motions must be carried out. The analysis for the wave equation in [2, 15] may be a guide for this type of generalisation of our analysis.

\section{Structure of the article}

The article is organised as follows: Section 2 provides a summary of the tensorial conventions to be used in the present article. Furthermore, in Subsection 2.2 a discussion of the procedure of how to coordinatise and introduce frame fields of the 3 -sphere, $\mathbb{S}^{3}$ is provided. Section 3 provides general remarks concerning perfect fluid cosmological models and a summary of the properties of the background solutions required in our subsequent analysis. These are summarised in Proposition 1. Section 4 gives a brief summary of the conformal Einstein field equations with matter. Section 5 provides a discussion of the Euler equations in the context of the conformal field equations. In Section 6 we discuss gauge considerations and the procedure leading to a hyperbolic reduction of the conformal field equations. The keys steps in this procedure have been discussed extensively elsewhere, so that this discussion is kept to a minimum. In particular, Subsection 6.2 provides a summary of the structural properties of the conformal evolution equations while Subsection 6.3 analyses the issue of the propagation of the constraints. Section 7 casts the FLRW background as a solution of the conformal field equations of Section 4 and analyses some of its properties. Finally, Section 8 is concerned with our main result - the existence and stability result for perfect fluid cosmologies with a de Sitter-like cosmological constant as given in Theorem 2 . 


\section{Notation and conventions}

\subsection{Index and curvature conventions}

Throughout this article we work with a spacetime $\left(\tilde{\mathcal{M}}, \tilde{g}_{\mu \nu}\right)$, where $\tilde{g}_{\mu \nu},(\mu, \nu=0,1,2,3)$ is a Lorentzian metric with signature $(+,-,-,-)$. We will denote by $\tilde{\nabla}$ the Levi-Civita connection of $\tilde{g}_{\mu \nu}$ - that is, the unique torsion-free connection that preserves the metric $\tilde{g}_{\mu \nu}$. In the sequel, $\tilde{R}_{\mu \nu \lambda \rho}, \tilde{R}_{\mu \nu}$ and $\tilde{R}$ will denote, respectively, the Riemann curvature tensor, the Ricci tensor and the Ricci scalar of the Levi-Civita connection $\tilde{\nabla}$. The conventions for the curvature used in this article are such that

$$
\tilde{R}_{\nu \lambda \rho}^{\mu} \xi^{\nu}=\left(\tilde{\nabla}_{\lambda} \tilde{\nabla}_{\rho}-\tilde{\nabla}_{\rho} \tilde{\nabla}_{\lambda}\right) \xi^{\mu}, \quad \tilde{R}_{\mu \nu}=\tilde{R}_{\nu \lambda \mu}^{\lambda}, \quad \tilde{R}=\tilde{R}_{\mu \nu} \tilde{g}^{\mu \nu}
$$

As a consequence of our signature conventions, then $\lambda<0$ corresponds to de Sitter-like values of the cosmological constant, while $\lambda>0$ corresponds to anti-de Sitter-like values. While $\mu, \nu, \ldots$ denote spacetime tensorial indices, $\alpha, \beta, \ldots$ denote spatial tensorial ones. Most of our discussion will be based on a frame formalism in which $i, j, \ldots$ denote spacetime indices ranging $0, \ldots, 3$. Similarly, $a, b, \ldots$ will denote spatial indices ranging $1,2,3$. Spinorial expressions and arguments will be used routinely, and we will follow the conventions of [21]. Consequently, the indices $A, B, \ldots$ will be spinorial ones.

\subsection{Coordinates and vector fields on the 3-sphere}

The present analysis will be concerned with spacetimes which are conformal to manifolds with topology $I \times \mathbb{S}^{3}$ where $I$ is an open interval on $\mathbb{R}$. In what follows, the manifold $\mathbb{S}^{3}$ will always be thought of as the following submanifold of $\mathbb{R}^{4}$ :

$$
\mathbb{S}^{3}=\left\{x^{\mathcal{A}} \in \mathbb{R}^{4} \mid\left(x^{1}\right)^{2}+\left(x^{2}\right)^{2}+\left(x^{3}\right)^{2}+\left(x^{4}\right)^{2}=1\right\} .
$$

The restrictions of the functions $x^{\mathcal{A}}, \mathcal{A}=1,2,3,4$ on $\mathbb{R}^{4}$ to $\mathbb{S}^{3}$ will again be denoted by $x^{\mathcal{A}}$. The vector fields

$$
\begin{aligned}
& c_{1} \equiv x^{1} \partial_{4}-x^{4} \partial_{1}+x^{2} \partial_{3}-x^{3} \partial_{2}, \\
& c_{2} \equiv x^{1} \partial_{3}-x^{3} \partial_{1}+x^{4} \partial_{2}-x^{2} \partial_{4}, \\
& c_{3} \equiv x^{1} \partial_{2}-x^{2} \partial_{1}+x^{3} \partial_{4}-x^{4} \partial_{3},
\end{aligned}
$$

on $\mathbb{R}^{4}$ are tangent to $\mathbb{S}^{3}$. In the sequel, they will always be considered as vectors on $\mathbb{S}^{3}$. The vector fields $\left\{c_{\underline{r}}\right\} \equiv\left\{c_{1}, c_{2}, c_{3}\right\}$ constitute a globally defined frame on $\mathbb{S}^{3}$ which is orthonormal with respect to the standard metric of $\mathbb{S}^{3}$. Moreover, the frame $\left\{c_{1}, c_{2}, c_{3}\right\}$ can be completed with a vector $c_{0}$ which is orthonormal to the standard metric on $I \times \mathbb{S}^{3},\left\{c_{\underline{s}}\right\} \equiv\left\{c_{0}, c_{1}, c_{2}, c_{3}\right\}$.

Let $\left(\mathcal{M}, g_{\mu \nu}\right)$ be a spacetime such that the manifold $\mathcal{M}$ is diffeomorphic to $\mathbb{R} \times \mathbb{S}^{3}$. A map $\Phi$ defined on an open subset $\mathcal{U} \subset \mathcal{M}$ will be said to be a cylinder map if it maps $\mathcal{U}$ diffeomorphically onto a set $I \times \mathbb{S}^{3}$, such that the sets $\Phi^{-1}\left(\{\tau\} \times \mathbb{S}^{3}\right)$ are spacelike Cauchy hypersurfaces of $\mathcal{M}$ and the curves $I \ni \tau \rightarrow \Phi^{-1}(\tau, p) \subset \mathcal{M}, p \in \mathbb{S}^{3}$ are timelike with respect to the metric $g_{\mu \nu}$. The cylinder map will be used to pull-back to $\mathcal{U}$ the coordinates $\left(\tau, x^{\mathcal{A}}\right) \equiv(\tau, \boldsymbol{x})$ in $I \times \mathbb{S}^{3}$. Furthermore, one can use $\Phi$ to pull-back to $\mathcal{U}$ the frame fields $c_{\underline{s}}$ defined in the previous paragraph. For simplicity of notation, such pull-back will be denoted again by $c_{\underline{s}}$.

\section{General remarks about FLRW cosmological models}

A cosmological model $\left(\tilde{\mathcal{M}}, \tilde{g}_{\mu \nu}, \tilde{u}^{\mu}\right)$ is a representation of the universe at a particular averaging scale. It is defined by a Lorentzian metric $\tilde{g}_{\mu \nu}$ on the manifold $\tilde{\mathcal{M}}$ and by a family of fundamental observers whose congruence of worldlines is represented by the timelike 4-velocity $\tilde{u}^{\mu}$ —usually taken to be the matter 4 -velocity. It is usually assumed that this congruence is expanding at 
some time. These assumptions together with a specification of the matter content are used to determine the dynamics of the universe. In what follows, it will be assumed that the interaction between geometry and matter is described by the Einstein field equations

$$
\tilde{R}_{\mu \nu}-\frac{1}{2} \tilde{R} \tilde{g}_{\mu \nu}+\lambda \tilde{g}_{\mu \nu}=\tilde{T}_{\mu \nu}
$$

and the energy-momentum conservation equation

$$
\tilde{\nabla}^{\mu} \tilde{T}_{\mu \nu}=0
$$

As already mentioned, the conventions for the cosmological constant $\lambda$ used in the present article are such that in vacuum, the case $\lambda<0$ describes a de Sitter-like spacetime, while the case $\lambda>0$ corresponds to an anti-de Sitter-like one.

Our discussion will be concerned with energy-momentum tensors of perfect fluids for which

$$
\tilde{T}_{\mu \nu}=(\tilde{\rho}+\tilde{p}) \tilde{u}_{\mu} \tilde{u}_{\nu}-\tilde{p} \tilde{g}_{\mu \nu},
$$

where $\tilde{\rho}, \tilde{p}$ and $\tilde{u}^{\mu}$ denote, respectively, the density, pressure and 4-velocity of the cosmological fluid. The fluid 4 -velocity $\tilde{u}^{\mu}$ is timelike and satisfies the normalisation condition $\tilde{u}_{\mu} \tilde{u}^{\mu}=1$.

The background solution whose non-linear stability will be considered in the present article belongs to the family of so-called Friedman-Lemaître-Robertson-Walker (FLRW) cosmological models. The FLRW models are homogeneous and isotropic. Their line element is usually given in the form

$$
\tilde{g}_{\mathscr{F}} \equiv \tilde{g}_{\mu \nu} \mathrm{d} x^{\mu} \mathrm{d} x^{\nu}=\mathrm{d} t^{2}-\frac{a^{2}(t)}{\left(1+\frac{1}{4} k r^{2}\right)^{2}}\left(\mathrm{~d} r^{2}+r^{2} \mathrm{~d} \theta^{2}+r^{2} \sin ^{2} \theta \mathrm{d} \varphi^{2}\right),
$$

where $a(t)$ is the so-called scale factor. This metric automatically defines a perfect fluid energymomentum tensor. When $k=0$ the spatial sections are flat, if $k<0$ the spatial sections have negative curvature, while if $k>0$, the spatial sections have positive curvature. The present analysis is concerned with FLRW cosmologies with spatial sections of positive curvature $(k=1)$ for which coordinates can be introduced such that:

$$
\tilde{g}_{\mathscr{F}}=\mathrm{d} t^{2}-a^{2}(t) \mathrm{d} \sigma^{2},
$$

with

$$
\mathrm{d} \sigma^{2} \equiv \mathrm{d} \psi^{2}+\sin ^{2} \psi \mathrm{d} \theta^{2}+\sin ^{2} \psi \sin ^{2} \theta \mathrm{d} \varphi^{2},
$$

the standard line element of $\mathbb{S}^{3}$ in polar coordinates. If the cosmological fluid satisfies the barotropic equation of state $\tilde{p}=(\gamma-1) \tilde{\rho}$, where $1 \leq \gamma \leq 2$ is a constant, then the evolution of the scale factor $a(t)$ is governed by the Friedmann equation:

$$
\frac{\dot{a}^{2}}{a^{2}}=-\frac{1}{3} \lambda-\frac{1}{a^{2}}+\frac{c}{a^{3 \gamma}}
$$

where $c$ is a constant. In what follows we will only be concerned with the case $\gamma=\frac{4}{3}$ corresponding to the so-called traceless perfect fluid (pure radiation). Furthermore, we assume $\lambda<0$. Equation (7) admits a static (i.e. time independent solution) in which the values of the scale factor and the cosmological constant are related by:

$$
a(t)=a_{0}=\text { constant }, \quad \lambda=\lambda_{0} \equiv \frac{3}{2} a_{0}^{-2} .
$$

In the dynamical case, under the assumptions $\gamma=\frac{4}{3}, \lambda<0$, the Friedmann equation (7) can be integrated explicitly - see e.g. [14. Different types of solutions are obtained, depending on the relative value of $\lambda$ with respect to $\lambda_{0}$ as given in equation (8), where $a_{0} \neq 0$ is now the value of the scale factor at some fiduciary time $t=t_{0} \neq 0$. The relevant properties for the analysis of these solutions are summarised in the following proposition: 
Proposition 1. For a FLRW cosmology with $k=1, \gamma=\frac{4}{3}$ and $\lambda<0, \lambda \neq \lambda_{0}$, the scale factor, $a(t)$, is a smooth, non-vanishing and monotonically increasing function for $t \in\left[t_{0}, \infty\right)$, with $t=t_{0}>0$ and $a_{0}=a\left(t_{0}\right)>0$. Furthermore,

$$
\int_{t_{0}}^{\infty} \frac{d s}{a(s)}<\infty
$$

and one has the limits

$$
a \rightarrow \infty, \quad \dot{a} / a \rightarrow \sqrt{-\frac{1}{3} \lambda}, \quad \ddot{a} / a \rightarrow-\frac{1}{3} \lambda .
$$

as $t \rightarrow \infty$. The pressure for these models is given by

$$
\tilde{\rho}=\tilde{\rho}_{0} a_{0}^{4} / a^{4},
$$

where $\tilde{\rho}_{0}=\tilde{\rho}\left(t_{0}\right)$. In particular, one has that $\tilde{\rho} \rightarrow 0$ as $t \rightarrow \infty$.

The proof of this proposition follows from direct inspection of the explicit solutions - see e.g. 14, page 78 .

Remark 3. A similar type of result can be obtained for FLRW models with $\gamma=\frac{4}{3}, \lambda \leq 0$ and $k=-1,0$. Again, see [14.

\section{The conformal field equations with matter}

The stability of the solutions to the Einstein equations described by the metric $\tilde{g}_{\mu \nu}$ corresponding to the line element (5) will be analysed in terms of a conformally related (unphysical) metric $g_{\mu \nu}$. This strategy leads to consider the conformal Einstein field equations. The idea of vacuum conformal Einstein field equations expressed in terms of the Levi-Civita connection $\nabla$ of the metric $g_{\mu \nu}$ and associated objects was originally introduced in [4, 5, 6]. The generalisation of these conformal equations to physical spacetimes containing matter was discussed in [10]. More recently, a more general type of vacuum conformal equations - the extended conformal Einstein field equations - expressed in terms of a Weyl connection $\hat{\nabla}$ has been introduced — see [11.

\subsection{Conformal rescalings}

All throughout we assume that the two metrics $\tilde{g}_{\mu \nu}$ and $g_{\mu \nu}$ are conformally related to each other via

$$
g_{\mu \nu}=\Theta^{2} \tilde{g}_{\mu \nu},
$$

where $\Theta$ is a non-negative scalar field - the conformal factor. The Christoffel symbols $\tilde{\Gamma}_{\mu}{ }^{\rho}{ }_{\nu}$ and $\Gamma_{\mu}^{\rho}{ }_{\nu}$ of the associated Levi-Civita connections $\tilde{\nabla}$ and $\nabla$ are related by

$$
\tilde{\Gamma}_{\mu}{ }^{\rho}{ }_{\nu}-\Gamma_{\mu}{ }^{\rho}{ }_{\nu}=S_{\mu \nu}{ }^{\rho \lambda} \Upsilon_{\lambda}
$$

where $\Upsilon_{\lambda}=\Theta^{-1} \nabla_{\lambda} \Theta$ and $S_{\mu \nu}{ }^{\rho \lambda}$ is the conformally invariant tensor

$$
S_{\mu \nu}^{\lambda \rho}=\delta_{\mu}^{\lambda} \delta_{\mu}^{\rho}+\delta_{\mu}{ }^{\rho} \delta_{\nu}{ }^{\lambda}-g_{\mu \nu} g^{\lambda \rho} .
$$

\subsection{Curvature tensors}

In a 4-dimensional spacetime the Schouten tensor, $P_{\mu \nu}$, of the connection $\nabla$ is defined by

$$
P_{\mu \nu}=\frac{1}{2} R_{\mu \nu}-\frac{1}{12} R g_{\mu \nu}
$$

The Schouten tensor of the connection $\tilde{\nabla}$ is defined by a similar expression involving the physical Ricci tensor and scalar. The tensors $\tilde{P}_{\mu \nu}$ and $P_{\mu \nu}$ are related by

$$
P_{\mu \nu}-\tilde{P}_{\mu \nu}=\nabla_{\mu} \Upsilon_{\nu}-\Upsilon_{\mu} \Upsilon_{\nu}+\frac{1}{2} g_{\mu \nu} \Upsilon_{\rho} \Upsilon^{\rho}
$$


We can thus decompose the Riemann curvature tensor, $R^{\mu}{ }_{\nu \lambda \rho}$, of the connection $\nabla$ into its irreducible parts as

$$
\begin{aligned}
R^{\mu}{ }_{\nu \lambda \rho} & =C^{\mu}{ }_{\nu \lambda \rho}+2 S_{\nu[\lambda}{ }^{\mu \sigma} P_{\rho] \sigma}, \\
& =C^{\mu}{ }_{\nu \lambda \rho}+2\left(g^{\mu}{ }_{[\lambda} P_{\rho] \nu}-g_{\nu[\lambda} P_{\rho]}{ }^{\mu}\right),
\end{aligned}
$$

where $C^{\mu}{ }_{\nu \lambda \rho}$ denotes the conformally invariant Weyl tensor.

As $\nabla$ is a Levi-Civita connection it satisfies the first and second Bianchi identities:

$$
\begin{aligned}
& R_{[\nu \lambda \rho]}^{\mu}=0, \\
& \nabla_{[\sigma} R^{\mu}{ }_{|\nu| \lambda \rho]}=0 .
\end{aligned}
$$

In our discussion of the conformal field equations with matter we will make use of the physical and unphysical Cotton-York tensors $\tilde{Y}_{\lambda \rho \nu}$ and $Y_{\lambda \rho \nu}$ given, respectively, by

$$
\tilde{Y}_{\lambda \rho \nu} \equiv \tilde{\nabla}_{\lambda} \tilde{P}_{\rho \nu}-\tilde{\nabla}_{\rho} \tilde{P}_{\lambda \nu}, \quad Y_{\lambda \rho \nu} \equiv \nabla_{\lambda} P_{\rho \nu}-\nabla_{\rho} P_{\lambda \nu} .
$$

The tensor $Y_{\lambda \rho \nu}$ appears in the once contracted Bianchi identity

$$
\nabla_{\mu} C^{\mu}{ }_{\nu \lambda \rho}=Y_{\lambda \rho \nu}
$$

Finally, it is noticed that the twice contracted Bianchi identity takes the form

$$
\nabla^{\nu} P_{\rho \nu}=\nabla_{\rho} P
$$

where $P=g^{\lambda \nu} P_{\lambda \nu}$.

\subsection{Frame and spinor formulations}

In what follows, consider a frame field $\left\{e_{i}\right\}, i=0, \ldots, 3$ which is orthogonal with respect to the metric $g_{\mu \nu}$. By construction one has that

$$
g_{\mu \nu} e_{i}^{\mu} e_{j}^{\nu}=\eta_{i j}, \quad \eta_{i j} \equiv \operatorname{diag}(1,-1,-1,-1) .
$$

In order to discuss the extended conformal Einstein field equations, it will be convenient to regard, for the moment, the connection $\nabla$ only as a metric connection with respect to $g_{\mu \nu}$-i.e. $\nabla_{\lambda} g_{\mu \nu}=0$. Under this assumption, the connection $\nabla$ could have torsion, and thus, it would not be a Levi-Civita connection. The connection coefficients, $\Gamma_{i}{ }^{k}{ }_{j}$, of $\nabla$ with respect to the frame $e_{k}$ are defined by the relation

$$
\nabla_{i} e_{j}=\Gamma_{i}{ }_{j}{ }_{j} e_{k} .
$$

As a consequence of having a metric connection, the connection coefficients satisfy

$$
\Gamma_{i}{ }_{j}{ }_{j} \eta_{k l}+\Gamma_{i}{ }^{k}{ }_{l} \eta_{k j}=0 .
$$

The torsion, $\Sigma_{i}{ }^{k}$, of the connection $\nabla$ is defined by

$$
\Sigma_{i}{ }_{j} e_{k} \equiv\left(\Gamma_{i}{ }_{j}{ }_{j}-\Gamma_{j}{ }^{k}{ }_{i}\right) e_{k}-\left[e_{i}, e_{j}\right] .
$$

If $\Sigma_{i}{ }_{j}{ }_{j}=0$ so that the connection $\nabla$ is the unique Levi-Civita connection of $g_{\mu \nu}$, the connection coefficients acquire the additional symmetry

$$
\Gamma_{i}{ }_{j}=\Gamma_{j}{ }^{k}{ }_{i} .
$$

Related to the $g$-orthonormal frame $e_{k}$ we will consider a normalised spinor dyad $\left\{\delta_{A}\right\}, A=$ 0,1 , such that

$$
e_{A A^{\prime}}=e_{k} \sigma_{A A^{\prime}}
$$

where $\sigma_{A A^{\prime}}^{k}$ are the constant van der Waerden symbols. 
In the sequel, a space spinor formalism will be introduced - see e.g. 25]. To this end, we consider a timelike spinor $\tau_{A A^{\prime}}$ which in terms of the dyad $\left\{\delta_{A}\right\}$ can be expressed as

$$
\tau^{A A^{\prime}}=\epsilon_{0}{ }^{A} \bar{\epsilon}_{0^{\prime}}{ }^{A^{\prime}}+\epsilon_{1}{ }^{A} \bar{\epsilon}_{1^{\prime}} A^{\prime} .
$$

In particular, one has the normalisation condition $\tau_{A A^{\prime}} \tau^{A A^{\prime}}=2$. The space spinor formalism allows to turn primed indices in spinorial expressions into unprimed ones by suitable contractions with $\tau_{A} A^{\prime}$ - see [10, 18, 19, 20] for more details. We simply recall that the space spinor decomposition of a spinor $u_{A A^{\prime}}$ is given by

$$
u_{A A^{\prime}}=\frac{1}{2} u \tau_{A A^{\prime}}-\tau_{A^{\prime}}^{Q} u_{Q A},
$$

where

$$
u \equiv u_{P P^{\prime}} \tau^{P P^{\prime}}, \quad u_{A B} \equiv \tau^{P^{\prime}}{ }_{(B} u_{A) P^{\prime}} .
$$

\subsection{The conformal field equations with tracefree matter}

In our subsequent discussion it will be convenient to distinguish between the geometric curvature $r^{k}{ }_{l i j}$-i.e. the expression of the curvature related to the connection coefficients $\Gamma_{i}{ }^{j}$ - and the algebraic curvature $R^{k}{ }_{l i j}$-i.e. the decomposition of the curvature in terms of irreducible components given by equation (12). One has that

$$
\begin{aligned}
& r^{k}{ }_{l i j} \equiv e_{i}\left(\Gamma_{j l}^{k}\right)-e_{j}\left(\Gamma_{i l}^{k}\right)-\Gamma_{m}{ }_{l}^{k}\left(\Gamma_{i}{ }_{j}^{m}-\Gamma_{j}{ }^{m}{ }_{i}\right)+\Gamma_{i{ }_{m}}^{k} \Gamma_{j}{ }^{m} l-\Gamma_{j}^{k}{ }_{m} \Gamma_{i}{ }^{m} l+\Sigma_{i}{ }_{j} \Gamma_{m}{ }^{k} l, \\
& R^{k}{ }_{l i j} \equiv C^{k}{ }_{l i j}+2\left(\delta^{k}{ }_{[i} P_{j] l}-\eta_{l[i} P_{j]}^{k}\right)=C^{k}{ }_{l i j}+2 S_{l[i}{ }^{k m} P_{j] m} .
\end{aligned}
$$

Following [13], in the sequel it will be convenient to introduce the variables

$$
\begin{aligned}
& d^{k}{ }_{l i j} \equiv \Theta^{-1} C^{k}{ }_{l i j}, \\
& d_{i} \equiv \nabla_{i} \Theta, \\
& s \equiv \frac{1}{4}\left(\nabla^{k} d_{k}+\Theta P_{k}^{k}\right) .
\end{aligned}
$$

Furthermore, we also consider the following zero quantities — cfr. [10]:

$$
\begin{aligned}
& \Sigma_{i j}^{l}{ }_{j} e_{l} \equiv\left(\Gamma_{i j}^{l}-\Gamma_{j i}^{l}\right) e_{l}-\left[e_{i}, e_{j}\right], \\
& \Xi^{k}{ }_{l i j} \equiv r^{k}{ }_{l i j}-R^{k}{ }_{l i j}, \\
& \Delta_{l i j} \equiv \nabla_{i} P_{j l}-\nabla_{j} P_{i l}-d_{k} d^{k}{ }_{l i j}-\Theta^{2} T_{i j l}, \\
& \Lambda_{l i j} \equiv \nabla_{k} d^{k}{ }_{l i j}-\Theta T_{i j l}, \\
& \delta_{k} \equiv d_{k}-\nabla_{k} \Theta, \\
& \delta_{i j} \equiv \nabla_{i} d_{j}+\Theta P_{i j}-s \eta_{i j}-\frac{1}{2} \Theta^{3} T_{i j}, \\
& \zeta_{k} \equiv \nabla_{k} s+d^{l} P_{k l}-\frac{1}{2} \Theta^{2} d^{l} T_{l k}, \\
& \zeta \equiv \lambda-6 \Theta s+3 d_{k} d^{k},
\end{aligned}
$$

where

$$
T_{k l} \equiv \Theta^{-2} \tilde{T}_{k l}, \quad T_{i j k} \equiv \Theta^{-2} \tilde{Y}_{i j k} .
$$

The interpretation of the zero quantities (19a)-(19d) is as follows: the zero quantity given by (19a) measures the torsion of the connection $\nabla$; that of (19b) relates the expression of the curvature of $\nabla$ with its decomposition in terms of irreducible components. Equations (19c) and (19d) measure the deviation from the fulfillment of the once contracted Bianchi identity. Finally, equations (19e), (19f) and (19g) bring into play the definitions (18b) and (18c) and give rise to differential conditions for the fields $\Theta, d_{i}$ and $s$.

The conformal Einstein field equations with matter are then given by

$$
\begin{aligned}
& \Sigma_{i}{ }^{k}{ }_{j} e_{k}=0, \quad \Xi^{k}{ }_{l i j}=0, \quad \Delta_{l i j}=0, \quad \Lambda_{l i j}=0, \\
& \delta_{k}=0, \quad \delta_{i j}=0, \quad \zeta_{k}=0, \quad \zeta=0 .
\end{aligned}
$$


These equations yield differential conditions for the frame coefficients $e_{i}$, the spin coefficients $\Gamma_{i}{ }^{j}{ }_{k}$, the components of the Schouten tensor $P_{i j}$, the rescaled Weyl tensor $d^{k}{ }_{l i j}$, the conformal factor $\Theta$, the 1-form $d_{i}$, and the scalar $s$, respectively. As discussed in e.g. [13, equation (19h) has the role of a constraint which holds by virtue of the other conformal field equations if it is satisfied on some initial hypersurface. It is noticed that as the torsion, $\Sigma_{i}{ }_{j}{ }_{j}$, is being introduced as a zero quantity, it can be consistently set to zero in the geometric curvature appearing in the definition for the zero quantity $\Xi^{k} l i j$ - equation (19b).

Equations (20a)-20b need to be complemented with the energy-momentum conservation equation (4). Its particular details will depend on the matter model under consideration.

Remark 4. Using a direct generalisation of the arguments presented in [5, 4] one can show that a solution to the conformal Einstein field equations with matter (20a)-(20b) and (4) give rise to a solution to the physical Einstein-matter system (3)-(4) — see also Theorem 3.1 in [6].

Remark 5. As a result of the conformal rescaling (9), the conformal equations (20a)-(20b) have a built-in conformal freedom which needs to be specified in order to deduce suitable evolution equations for the conformal fields. Further gauge freedom in equations (20a $-(20 \mathrm{~b})$ is concerned with the partial specification of the frame $e_{k}$ and the choice of coordinates. These will be specified by the choice of suitable gauge source functions.

\section{Perfect fluids in the context of the conformal approach}

In this section we present a discussion of the relativistic equations describing a perfect fluid which is geared towards our particular applications.

\subsection{The energy-momentum tensor and its transformation rules}

Given the spacetime $\left(\tilde{\mathcal{M}}, \tilde{g}_{\mu \nu}\right)$, the energy-momentum tensor for a perfect fluid with 4 -velocity $\tilde{u}^{i}$, pressure $\tilde{p}$, and density $\tilde{\rho}$ has the form

$$
\tilde{T}_{\mu \nu}=(\tilde{\rho}+\tilde{p}) \tilde{u}_{\mu} \tilde{u}_{\nu}-\tilde{p} \tilde{g}_{\mu \nu} .
$$

In order to perform a discussion of the perfect fluid in the conformally rescaled (unphysical) spacetime one introduces unphysical versions of the physical fields. More precisely, one defines

$$
T_{\mu \nu} \equiv \Theta^{-2} \tilde{T}_{\mu \nu}, \quad u_{\mu} \equiv \Theta \tilde{u}_{\mu}, \quad \rho \equiv \Theta^{-4} \tilde{\rho}, \quad p \equiv \Theta^{-4} \tilde{p} .
$$

Following the approach used in the discussion of geometric fields, we will work directly with the frame components $T_{i j} \equiv e_{i}{ }^{\mu} e_{j}{ }^{\nu} T_{\mu \nu}$ and $u_{i} \equiv e_{i}{ }^{\mu} u_{\mu}$ with respect to a $g$-orthonormal frame $e_{i}$. Thus

$$
T_{i j}=(\rho+p) u_{i} u_{j}-p \eta_{i j} .
$$

We observe that $\tilde{g}(\tilde{u}, \tilde{u})=1$ implies that $g(u, u)=1$. Now, using $u_{i}=\eta_{i j} u^{j}, u^{i}=\eta^{i j} u_{j}$, our signature convention implies

$$
u^{0}=u_{0}, \quad u^{a}=-u_{a}, \quad a=1,2,3 .
$$

A computation using the standard transformation rules for the covariant derivatives of conformally rescaled metrics yields

$$
\eta^{i j} \nabla_{i} T_{j k}=\Theta^{-4} \tilde{\eta}^{i j} \tilde{\nabla}_{i} \tilde{T}_{j k}-\Theta^{-5} \tilde{\nabla}_{k} \Theta \tilde{\eta}^{i j} \tilde{T}_{i j}
$$

Consequently, the (physical) equation for the conservation of energy-momentum

$$
\tilde{\nabla}^{j} \tilde{T}_{i j}=0
$$

implies an analogous equation

$$
\nabla^{j} T_{i j}=0
$$


for the (unphysical) conformally rescaled spacetime only if the energy-momentum tensor $\tilde{T}_{i j}$ is tracefree - see [10]. Notice that $\tilde{T} \equiv \tilde{\eta}^{i j} \tilde{T}_{i j}=0$ if and only if $T \equiv \eta^{i j} T_{i j}=0$. A quick computation shows that for a perfect fluid the tracefreeness of the energy-momentum tensor implies $\rho-3 p=0$ -in other words $\gamma=\frac{4}{3}$. Hence

$$
p=\frac{1}{3} \rho, \quad \tilde{p}=\frac{1}{3} \tilde{\rho} .
$$

This class of perfect fluids is usually referred to as pure radiation.

In the present article, our analysis will be restricted to the case of tracefree perfect fluids. The unphysical energy-momentum tensor for this class of perfect fluids reduces to

$$
T_{i j}=\frac{4}{3} \rho u_{i} u_{j}-\frac{1}{3} \rho \eta_{i j} .
$$

As a consequence of the definition of the 4 -velocity $u^{i}$ it follows that

$$
\begin{aligned}
& \eta_{i j} u^{i} u^{j}=u_{k} u^{k}=u_{0} u^{0}+u_{a} u^{a}=1, \\
& \nabla_{k} u^{0}=-\frac{u_{a}}{u_{0}} \nabla_{k} u^{a}, \\
& \nabla_{l} \nabla_{k} u^{0}=-\frac{u_{a}}{u_{0}} \nabla_{l} \nabla_{k} u^{a}-\frac{1}{u_{0}} \nabla_{l} u_{a} \nabla_{k} u^{a}-\frac{u_{a} u_{b}}{u_{0}^{3}} \nabla_{l} u^{b} \nabla_{k} u^{a} .
\end{aligned}
$$

These identities will be used to rewrite the component $u^{0}$ and its derivatives in terms of the spatial components $u^{a}$ and their derivatives. This procedure will be central for the construction of a symmetric hyperbolic system for the matter variables. It is also noticed that equation (24a) implies

$$
u^{k} \nabla_{k}\left(u_{i} u^{i}\right)=0
$$

This expression shows that if $u_{i} u^{i}=1$ at some point in a fluid flow line, then $u_{i} u^{i}=1$ in the whole flow line.

\subsection{The energy conservation equation and the equations of motion}

A direct computation shows that the conservation equation (22) implies

$$
Z_{j} \equiv \frac{4}{3}\left(u_{j} u^{i} \nabla_{i} \rho+\rho u_{j} \nabla_{i} u^{i}+\rho u^{i} \nabla_{i} u_{j}\right)-\frac{1}{3} \nabla_{j} \rho=0 .
$$

This equation can be split into components parallel and orthogonal to $u^{i}$ :

$$
\begin{aligned}
& u^{i} Z_{i}=u^{i} \nabla_{i} \rho+\frac{4}{3} \rho \nabla_{i} u^{i}=0, \\
& \gamma_{j}{ }^{i} Z_{i}=\frac{4}{3} \rho u^{i} \nabla_{i} u_{j}+\frac{1}{3} u_{j} u^{i} \nabla_{i} \rho-\frac{1}{3} \nabla_{j} \rho=0,
\end{aligned}
$$

where

$$
\gamma_{i j} \equiv \eta_{i j}-u_{i} u_{j}
$$

These equations are the conformal versions of the equation of energy conservation and the equations of motion - see e.g. [3. It is noticed that equations (26a) and (26b) can be combined to give

$$
\nabla_{j} \rho=4 \rho u^{i} \nabla_{i} u_{j}-\frac{4}{3} \rho u_{j} \nabla_{i} u^{i} .
$$

This equation will be used in the sequel to eliminate the gradient of the unphysical density from certain expressions.

\subsection{A symmetric hyperbolic system for the fluid fields}

The equations of conservation of energy and motion will be used to construct a symmetric hyperbolic system of evolution equations for the unphysical density $\rho$ and the spatial components of the unphysical velocity $u^{a}$. The procedure used here follows the presentation given in [3]. In the sequel, it should be understood that, consequently with equation (24a),

$$
u_{0}=u^{0}=\sqrt{1-u_{a} u^{a}} .
$$


Substituting identity (24b) into (26a) gives

$$
\frac{3}{16 \rho^{2}}\left(u^{0} \nabla_{0} \rho+u^{a} \nabla_{a} \rho\right)+\frac{1}{4 \rho}\left(\nabla_{a} u^{a}-\frac{u_{a}}{u_{0}} \nabla_{0} u^{a}\right)=0,
$$

where the extra factor $1 / 4 \rho$ has been included to ensure symmetric hyperbolicity. Similarly, from equation (26b) one deduces

$$
\begin{aligned}
& \gamma^{0 k} Z_{k}=u^{k} \nabla_{k} u^{0}+\frac{1}{4 \rho} u^{0} u^{k} \nabla_{k} \rho-\frac{1}{4 \mu} \eta^{0 k} \nabla_{k} \rho=0, \\
& \gamma^{a k} Z_{k}=u^{k} \nabla_{k} u^{a}+\frac{1}{4 \rho} u^{a} u^{k} \nabla_{k} \rho-\frac{1}{4 \rho} \eta^{a k} \nabla_{k} \rho=0 .
\end{aligned}
$$

In order to obtain suitable evolution equations for the spatial components of the 4 -velocity, we consider the combination

$$
\varsigma^{a} \equiv \frac{u^{a}}{u^{0}} \gamma^{0 k} Z_{k}-\gamma^{a k} Z_{k}=0
$$

or equivalently

$$
\varsigma^{a}=\left(u^{i} \nabla_{i} u^{a}+\frac{u^{a} u^{i} u_{c}}{u^{0} u_{0}} \nabla_{i} u^{c}\right)+\frac{1}{4 \rho}\left(\frac{u^{a}}{u^{0}} \eta^{0 k} \nabla_{k} \rho-\eta^{a k} \nabla_{k} \rho\right)=0 .
$$

A direct inspection shows that:

Lemma 1. Equations (28) and (29) constitute a symmetric hyperbolic system for the fields $\rho$ and $u^{a}$.

One also has that:

Lemma 2. A solution $\left(\rho, u^{a}\right)$ to the evolution equations (28) and (29) implies a solution $\left(\rho, u_{0}, u^{a}\right)$ to equation (25) with $u_{0}=\sqrt{1-u_{a} u^{a}}$.

Proof. We need to show $Z^{j}=0$. The definition of $u_{0}$ implies that (24b) and (24c) hold. Now, given a solution to (28) and (29), the right hand side of (28) can be rewritten so as to yield $u^{j} Z_{j}=0$. Substitution into the left hand side of (29) gives

$$
\frac{u^{a}}{u^{0}} Z^{0}-Z^{a}=0
$$

Contracting with $u_{a}$ and using (24a), as well as $u_{0} \geq 1$ gives first $Z^{0}=0$ and then $Z^{a}=0$. Hence a solution to (28) and (29) satisfies (25).

Remark 6. Let $u^{A A^{\prime}}$ denote the spinorial counterpart of the 4 -velocity vector $u^{\mu}$. The spinor $u^{A A^{\prime}}$ can be split using the spinor $\tau^{A A^{\prime}}$ as done in (17). This implies

$$
u=\sqrt{2} u_{0}=\sqrt{2} u^{0}, \quad u_{A B}=\sigma^{a}{ }_{A B} u_{a},
$$

where $\sigma_{A B}^{a}$ denote the spatial Infeld-van der Waerden symbols. It follows that (25) implies a symmetric hyperbolic system for the spinorial components $u$ and $u_{A B}$. The explicit form of these equations will not be required in our subsequent analysis.

\subsection{The Cotton-York tensor of a traceless perfect fluid spacetime}

The matter field quantities feedback into the geometric part of the conformal field equation through the physical Cotton-York tensor $\tilde{Y}_{\mu \nu \lambda}$. In what follows, the latter is expressed in terms of tensors, however the frame and spinor component versions are easily derived from these equations.

For a tracefree energy momentum tensor the physical Schouten tensor is given by $\tilde{P}_{i j}=\frac{1}{2} \tilde{T}_{i j}$ so that

$$
\tilde{Y}_{i j k}=\tilde{\nabla}_{[i} \tilde{T}_{j] k}
$$


Rewriting this expression in terms of unphysical quantities one obtains for $T_{i j k}=\Theta^{-2} \tilde{Y}_{i j k}$ that

$$
T_{i j k}=\nabla_{[i} T_{j] k}+\Upsilon_{[i} T_{j] k}+g_{k[i} T_{j] l} \Upsilon^{l} .
$$

The last two terms in this expression are polynomial in $\rho$ and the components $u^{i}$. The first term, however, contains derivatives of $u^{i}$ and $\rho$ that would enter the principal part of the Cotton-York and Bianchi equations. The fluid equations cannot be used to eliminate these derivatives.

In order to get around this difficulty, we introduce new variables $\rho_{k}$ and $u_{i j}$ and corresponding zero quantities $q_{k}$ and $y_{i j}$ via

$$
q_{k} \equiv \rho_{k}-\nabla_{k} \rho, \quad y_{i j} \equiv u_{i j}-\nabla_{i} u_{j} .
$$

Observe that if $q_{k}=0$ and $y_{i j}=0$, one then has that $u_{i j} u^{j}=0$ and $y_{i j} u^{j}=0$, so that one can write

$$
u_{i 0}=-\frac{u^{a}}{u_{0}} u_{i a}, \quad y_{i 0}=-\frac{u^{a}}{u_{0}} y_{i a} .
$$

Furthermore, from $u_{i j} u^{j}=0$, it also follows that

$$
\begin{aligned}
& \nabla_{k} u_{i}{ }^{0}=-\frac{u_{a}}{u_{0}} \nabla_{k} u_{i}{ }^{a}+\frac{u_{k}{ }^{0}}{u_{0}}\left(y_{i 0}-u_{i 0}\right)+\frac{u_{k}{ }^{a}}{u_{0}}\left(y_{i a}-u_{i a}\right), \\
& u^{j} \nabla_{i} u_{k j}=u_{k j} y_{i}{ }^{j}-u_{k j} u_{i}{ }^{j} .
\end{aligned}
$$

Finally, if $q_{k}=0, y_{i j}=0$, then the first term of $T_{i j k}$ can be written as

$$
\begin{aligned}
\nabla_{[i} T_{j] k} & =\frac{4}{3}\left(\nabla_{[i} \rho u_{j]} u_{k}+\rho \nabla_{[i} u_{j]} u_{k}+\rho u_{[j} \nabla_{i]} u_{k}\right)-\frac{1}{3} \nabla_{[i} \rho \eta_{j] k}, \\
& =\frac{4}{3}\left(\rho_{[i} u_{j]} u_{k}+\rho u_{[i j]} u_{k}+\rho u_{[j} u_{i] k}\right)-\frac{1}{3} \rho_{[i} \eta_{j] k} .
\end{aligned}
$$

\subsubsection{A symmetric hyperbolic system for $\rho_{k}$ and $u_{k}{ }^{a}$}

The evolution equations for $\rho$ and $u^{a}$ are derived from equation (25). Taking derivatives of (25) and commuting them gives:

$$
\begin{aligned}
0=\nabla_{k} Z_{j}= & \frac{4}{3}\left(u_{j} u^{i} \nabla_{i} \nabla_{k} \rho+\rho u_{j} \nabla_{i} \nabla_{k} u^{i}+\rho u^{i} \nabla_{i} \nabla_{k} u_{j}\right)-\frac{1}{3} \nabla_{j} \nabla_{k} \rho \\
& +\frac{4}{3}\left(u_{j} u^{i} \Sigma_{k}{ }_{k} \nabla_{l} \rho+\rho u_{j} r_{k i}{ }^{i}{ }_{l} u^{l}-\rho u^{i} r_{k i}{ }^{l} u_{l}+\nabla_{k} u_{j} u^{i} \nabla_{i} \rho+\nabla_{k} \rho u_{j} \nabla_{i} u^{i}\right. \\
& \left.+\nabla_{k} \rho u^{i} \nabla_{i} u_{j} u_{j} \nabla_{k} u^{i} \nabla_{i} \rho+\rho \nabla_{k} u_{j} \nabla_{i} u^{i}+\rho \nabla_{k} u^{i} \nabla_{i} u_{j}\right)-\frac{1}{3} \Sigma_{k}{ }_{k} \nabla_{l} \rho, \\
= & \frac{4}{3}\left(u_{j} u^{i} \nabla_{i} \nabla_{k} \rho+\rho u_{j} \nabla_{i} \nabla_{k} u^{i}+\rho u^{i} \nabla_{i} \nabla_{k} u_{j}\right)-\frac{1}{3} \nabla_{j} \nabla_{k} \rho+V_{k j},
\end{aligned}
$$

where all terms with at most one derivative of $\rho$ or $u^{k}$ have been gathered in $V_{k j}$. In view of this discussion, in the sequel we will consider the field equation for $\rho_{k}$ and $u_{i j}$ given by the following zero quantity:

$$
Z_{k j} \equiv \frac{4}{3}\left(u_{j} u^{i} \nabla_{i} \rho_{k}+\rho u_{j} \nabla_{i} u_{k}^{i}+\rho u^{i} \nabla_{i} u_{k j}\right)-\frac{1}{3} \nabla_{j} \rho_{k}+W_{k j}=0,
$$

with

$$
\begin{gathered}
W_{k j} \equiv \frac{4}{3}\left(u_{j} u^{i} \Sigma_{k}{ }_{i} \rho_{l}+\rho u_{j} r_{k i}{ }_{l}^{i} u^{l}-\rho u^{i} r_{k i}{ }^{l}{ }_{j} u_{l}+u_{k j} u^{i} \rho_{i}+\rho_{k} u_{j} u_{i}{ }^{i}+\rho_{k} u^{i} u_{i j}\right. \\
\left.+u_{j} u_{k}{ }^{i} \rho_{i}+\rho u_{k j} u_{i}{ }^{i}+\rho u_{k}{ }^{i} u_{i j}\right)-\frac{1}{3} \Sigma_{k j}{ }^{l} \rho_{l} .
\end{gathered}
$$

From the equation $Z_{i j}=0$ one derives, in analogy to (26b) and (26a), that

$$
\begin{aligned}
& u^{j} Z_{k j} \equiv u^{i} \nabla_{i} \rho_{k}+\frac{4}{3} \rho \nabla_{i} u_{k}{ }^{i}+X_{k}=0, \\
& \gamma_{j}^{l} Z_{k l} \equiv \frac{4}{3} \rho u^{i} \nabla_{i} u_{k j}+\frac{1}{3} u_{j} u^{i} \nabla_{i} \rho_{k}-\frac{1}{3} \nabla_{j} \rho_{k}+X_{k j}=0,
\end{aligned}
$$

where (32b) has been used and

$$
\begin{aligned}
& X_{k} \equiv \frac{4}{3} \rho u^{i}\left(u_{k j} y_{i}{ }^{j}-u_{k j} u_{i}{ }^{j}\right)+u^{j} W_{k j} \\
& X_{k j} \equiv-\frac{4}{3} \rho u_{j} u^{i}\left(u_{k l} y_{i}{ }^{l}-u_{k l} u_{i}{ }^{l}\right)+\gamma_{j}{ }^{l} W_{k l} .
\end{aligned}
$$


Finally, we rewrite (35a) in the form

$$
\frac{3}{16 \rho^{2}}\left(u^{0} \nabla_{0} \rho_{k}+u^{a} \nabla_{a} \rho_{k}\right)+\frac{1}{4 \rho}\left(\nabla_{a} u_{k}^{a}-\frac{u_{a}}{u_{0}} \nabla_{0} u_{k}^{a}\right)+\hat{X}_{k}=0
$$

where

$$
\hat{X}_{k}=\frac{3}{16 \rho^{2}} X_{k}+\frac{u^{i}}{4 \rho}\left(\frac{u_{k}^{0}}{u_{0}}\left(y_{i 0}-u_{i 0}\right)+\frac{u_{k}^{a}}{u_{0}}\left(y_{i a}-u_{i a}\right)\right) .
$$

Similarly, the combination

$$
\frac{u^{a}}{u_{0}} \gamma^{0 l} Z_{k l}-\gamma^{a l} Z_{k l}
$$

leads to the evolution equation

$$
\left(u^{i} \nabla_{i} u_{k}{ }^{a}+\frac{u^{a} u^{i} u_{c}}{u^{0} u_{0}} \nabla_{i} u_{k}^{c}\right)+\frac{1}{4 \rho}\left(\frac{u^{a}}{u^{0}} \eta^{0 l} \nabla_{l} \rho_{k}-\eta^{a l} \nabla_{l} \rho_{k}\right)+\hat{X}_{k}{ }^{a}=0 .
$$

where $\hat{X}_{k}{ }^{a}$ is a combination of

$$
\frac{u^{a}}{u_{0}} \gamma^{0 l} X_{k l}-\gamma^{a l} X_{k l}
$$

and terms from expression (32a).

In analogy to Lemma 1 one can readily verify that:

Lemma 3. If $y_{i j}=0$, then equations (36) and (37) constitute a symmetric hyperbolic system for the fields $\rho_{k}$ and $u_{k}{ }^{a}$.

A similar argument to the one leading to Lemma 2 yields:

Lemma 4. Let $y_{i j}=0$. A solution $\left(\rho_{k}, u_{k}{ }^{a}\right)$ to the evolution equations (36) and (37) implies a solution to equation (34).

\subsubsection{The subsidiary equations for the fluid variables}

In this section we derive evolution equations for the zero quantities $q_{i}$ and $y_{i j}$. These subsidiary equations will be of relevance in the discussion of the propagation of the constraints - see Section 6.3 .

Subtracting equation (33) from equation (34) gives

$$
Q_{k j} \equiv \frac{4}{3}\left(u_{j} u^{i} \nabla_{i} q_{k}+\rho u_{j} \nabla_{i} y_{k}{ }^{i}+\rho u^{i} \nabla_{i} y_{k j}\right)-\frac{1}{3} \nabla_{j} q_{k}+W_{k j}-V_{k j}=0 .
$$

Now, using substitutions like

$$
u_{i j} \rho_{k}-\nabla_{i} u_{j} \nabla_{k} \rho=u_{i j} q_{k}+y_{i j} \rho_{k}-y_{i j} q_{k}
$$

one can deduce that all individual terms in sums of $W_{k j}-V_{k j}$ in equation (38) contain at least one zero quantity. Repeating the discussion for the evolution equations for $\left(\rho_{k}, u_{i j}\right)$ with $\left(q_{k}, y_{i j}\right)$ one finds that

$$
\begin{aligned}
& \frac{3}{16 \rho^{2}}\left(u^{0} \nabla_{0} q_{k}+u^{a} \nabla_{a} q_{k}\right)+\frac{1}{4 \rho}\left(\nabla_{a} y_{k}{ }^{a}-\frac{u_{a}}{u_{0}} \nabla_{0} y_{k}{ }^{a}\right)+Y_{k}=0, \\
& \left(u^{i} \nabla_{i} y_{k}{ }^{a}+\frac{u^{a} u^{i} u_{c}}{u^{0} u_{0}} \nabla_{i} y_{k}{ }^{c}\right)+\frac{1}{4 \rho}\left(\frac{u^{a}}{u^{0}} \eta^{0 l} \nabla_{l} q_{k}-\eta^{a l} \nabla_{l} q_{k}\right)+Y_{k}{ }^{a}=0,
\end{aligned}
$$

where all terms in $Y_{k}$ and $Y_{k}{ }^{a}$ contain zero quantities. The evolution equations (39) and (40) constitute a symmetric hyperbolic system for the independent components of $q_{k}$ and $y_{i j}$. 


\subsubsection{Final remarks}

As a consequence of the analysis in the previous subsections one has that the components $T_{i j k}$ of the tensor $T_{\mu \nu \lambda}$ with respect to the frame $e_{i}$ are polynomial expressions of the unknowns $\rho, \rho_{j}$, $u_{i}$ and $u_{i j}$. If desired, the dependence with respect to $\rho_{k}$ can be eliminated using equation (27). As long as $\rho \neq 0$, the fields $\rho, \rho_{j}, u_{i}$ and $u_{i j}$ satisfy symmetric hyperbolic equations 1 . Finally, it is noticed that if the fields $\rho, \rho_{j}, u_{i}$ and $u_{i j}$ are regular at the points where $\Theta=0$, then $T_{i j k}$ is also regular - and consequently, also equations (19c) and (19d) are formally regular.

\section{A symmetric hyperbolic reduction of the conformal field equations}

In the previous section it has been shown how the equations of motion for the fluid variables and their derivatives lead to a system of symmetric hyperbolic equations independently of geometric gauge considerations. The purpose of this section is to briefly discuss a reduction procedure for the geometric unknowns. Our treatment is inspired on the one given in [10, but it also combines ideas from [9, 19].

\subsection{Gauge freedom}

As mentioned previously, the conformal Einstein field equations (20a) and (20b) are endowed with three classes of gauge freedom: conformal, coordinate and frame. In what follows, we briefly discuss a procedure for fixing this freedom.

\subsubsection{Conformal gauge freedom}

As already mentioned, the conformal Einstein field equations (20a) and (20b) admit certain freedom in the specification of the representative, $g_{\mu \nu}$, of the conformal class $\left[\tilde{g}_{\mu \nu}\right]$ which will be used as the unphysical metric - see e.g. [13] and references therein for more details.

Assuming for a moment that one has a solution to the conformal Einstein field equations with matter, it follows then by contraction of indices in equation (11) together with the tracefreeness of the energy momentum tensor that

$$
\nabla_{k} \nabla^{k} \Theta=\Theta P_{k}^{k}
$$

As discussed in, e.g. [13], this equation can always be solved locally so that the condition

$$
P \equiv P_{k}^{k}=-1,
$$

holds. This condition fixes the conformal freedom in the equations (20a) and (20b).

\subsubsection{Coordinate and gauge freedom}

In order to fix the coordinate and frame gauge freedom, we make use of the notion of gauge source functions - see [7, 10, 13]. The gauge source freedoms will be chosen so as to render symmetric hyperbolic evolution equations for the geometric unknowns.

In what follows we encounter equations of the form

$$
2 \nabla_{[i} M_{j] \ldots}=N_{[i j] \cdots}
$$

where the dots denote an arbitrary set of indices - cfr. equations (19a)-119c). The spinorial equivalents of the above equation are given by

$$
\nabla_{A\left(A^{\prime}\right.} M^{A}{ }_{\left.B^{\prime}\right) \cdots}=N_{A\left(A^{\prime}\right.}{ }^{A}{ }_{\left.B^{\prime}\right) \cdots}
$$

\footnotetext{
${ }^{1}$ An alternative evolution system which is valid even if $\rho=0$ can be obtained using the ideas of [22].
} 
and its complex conjugate. Now, an equation of the form

$$
\nabla_{A A^{\prime}} M^{A}{ }_{B^{\prime} \ldots}=N_{A A^{\prime}}{ }^{A}{ }_{B^{\prime} \cdots}
$$

is well known to imply a symmetric hyperbolic evolution system for the independent components of $M_{A A^{\prime}}$-see e.g. [7]. Note, however, that equation (42) contains no information about the skew term

$$
\nabla_{A\left[A^{\prime}\right.} M^{A}{ }_{\left.B^{\prime}\right] \ldots}=\frac{1}{2} \epsilon_{A^{\prime} B^{\prime}} \nabla^{C C^{\prime}} M_{C C^{\prime} \ldots}=F \ldots
$$

which can be specified arbitrarily. Thus, by adding (44) with a convenient choice of a gauge source function, $F \ldots$, to (42) one obtains an equation of the form of (43), from where a symmetric hyperbolic system can be extracted — see e.g. [7, 13].

The previous discussion will be implemented in the field equations (19a), (19b) and (19c). These equations provide differential conditions for the fields $e_{i}^{\mu}, P_{i j}$ and $\Gamma_{i}{ }^{j}{ }_{k}$. Let $e_{A A^{\prime}}^{\mu}, P_{A A^{\prime} B B^{\prime}}$ and $\Gamma_{A A^{\prime}}{ }^{B B^{\prime}} C C^{\prime}$ denote the spinorial counterparts of these fields. As a consequence of the metricity of the connection, instead of working with $\Gamma_{A A^{\prime}} B B^{\prime} C_{C C^{\prime}}$, we will consider a spinorial field $\Gamma_{A A^{\prime} B C}$ such that

$$
\Gamma_{A A^{\prime}}{ }^{B B^{\prime}}{ }_{C C^{\prime}}=\Gamma_{A A^{\prime}}{ }^{B}{ }_{C} \epsilon_{C^{\prime}} B^{\prime}+\bar{\Gamma}_{A^{\prime} A^{B^{\prime}}}{ }_{C^{\prime}} \epsilon_{C}{ }^{B} .
$$

For convenience, define the gauge source functions

$$
\begin{aligned}
& F^{\mu} \equiv \nabla^{A A^{\prime}} e_{A A^{\prime}}^{\mu}, \\
& F_{(B C)} \equiv \nabla^{A A^{\prime}} \Gamma_{A A^{\prime} B C}, \\
& F_{B B^{\prime}} \equiv \nabla^{A A^{\prime}} P_{A A^{\prime} B B^{\prime}}=\nabla_{B B^{\prime}} P,
\end{aligned}
$$

where the second equality in the definition of $F_{B B^{\prime}}$ follows from the twice contracted Bianchi identity for the unphysical connection $\nabla$. Motivated by their value in the reference solution (the conformal FLRW solution) the gauge source functions will be fixed by the conditions

$$
F^{\mu}=0, \quad F_{(B C)}=0, \quad F_{B B^{\prime}}=0 .
$$

Notice, in particular, that the last condition is consistent with the conformal gauge condition (41). As discussed in [7] - see also [10] - a particular choice of the coordinate and frame gauge functions $F^{\mu}$ and $F_{(B C)}$ fixes the coordinates and frame 2 .

The spacetimes to be considered in the present analysis have the topology of $\mathbb{R} \times \mathbb{S}^{3}$. Given an initial manifold $\mathcal{S}$ for the spacetime, then there is a diffeomorphism $\Phi: \mathcal{S} \rightarrow \mathbb{S}^{3}$ which allows to pull-back coordinates from $\mathbb{S}^{3}$ to $\mathcal{S}$. These coordinates on the initial manifold $\mathcal{S}$ will be used as the initial value of the spatial part of the spacetime coordinates. The time coordinate will be set initially to zero. The initial value of the frame $e_{i}$ is set by choosing on $\mathcal{S}$ some arbitrary orthonormal spatial frame $e_{a}$ (with respect to the 3 -metric of $\mathcal{S}$ ). The $e_{0}$ vector is set to coincide initially with the (spacetime) normal to $\mathcal{S}$.

\subsection{The evolution equations}

The hyperbolic reduction of the matter variables has already been discussed in Sections 5.3 and 5.4.1. In what concerns the evolution equations for the geometric variables, we follow the procedure indicated in [10]. This consists of a rewriting the spinorial version of the conformal field equations (20a)-(20b) in terms of space spinors so that the resulting equations contain only unprimed indices. In order to encompass the full information of the field equations, one has to include into the set of equations their Hermitian conjugates. If the fields and equations are then decomposed into their irreducible parts, then the equations split in a natural way into symmetric hyperbolic evolution and constraint equations. This procedure is straightforward, but involves lengthy computations, most of which can now be implemented in a computer algebra system like the suite xAct for Mathematica 3 .

\footnotetext{
${ }^{2}$ The gauge source functions $F^{\mu}$ and $F_{(B C)}$ imply, respectively, wave equations for the coordinates and a semi-linear equation for the frame components. These equations can be solved locally.

${ }^{3}$ See www.xAct.es.
} 
The required evolution equations have already been deduced in [10]. Their detailed form will not be required here. Instead we present a summary of their key structural properties. In what follows let

$$
\begin{aligned}
& \boldsymbol{v} \equiv\left(\Theta, d_{A B}, s, e_{\frac{r}{A} B}, \Gamma_{A B C D}, P_{A B C D}\right), \\
& \boldsymbol{\phi} \equiv \phi_{A B C D}, \\
& \varrho \equiv\left(\rho, u_{(A B)}\right), \\
& \boldsymbol{\psi} \equiv\left(\rho_{A B}, u_{A B(C D)}\right),
\end{aligned}
$$

where only the independent irreducible components of the spinors are taken into account. In terms of these objects, the evolution equations have the form

$$
\begin{aligned}
& \partial_{0} \boldsymbol{v}+\boldsymbol{A}_{[\boldsymbol{r}]}^{\underline{r}}(\boldsymbol{v}) c_{\underline{r}} \boldsymbol{v}=\boldsymbol{B}_{[\boldsymbol{v}]}(\boldsymbol{v}) \boldsymbol{v}+\boldsymbol{M}_{[\boldsymbol{v}]}(\boldsymbol{v}, \phi, \varrho, \boldsymbol{\psi}), \\
& \left(\sqrt{2} \boldsymbol{E}+\boldsymbol{A}_{[\phi]}^{\underline{0}}(\boldsymbol{v})\right) \partial_{0} \boldsymbol{\phi}+\boldsymbol{A}_{[\boldsymbol{\phi}]}^{\underline{r}}(\boldsymbol{v}) c_{\underline{r}} \boldsymbol{\phi}=\boldsymbol{B}_{[\phi]}(\boldsymbol{v}) \boldsymbol{\phi}+\boldsymbol{M}_{[\boldsymbol{\phi}]}(\boldsymbol{v}, \boldsymbol{\psi}, \varrho), \\
& \boldsymbol{A}_{[\boldsymbol{\varrho}]}^{\underline{0}}(\boldsymbol{v}, \varrho) \partial_{0} \varrho+\boldsymbol{A}_{[\varrho]}^{\underline{r}}(\boldsymbol{v}, \varrho) c_{\underline{r}} \boldsymbol{\varrho}=\boldsymbol{B}_{[\varrho]}(\boldsymbol{v}) \boldsymbol{\varrho}, \\
& \boldsymbol{A}_{[\boldsymbol{}}^{\underline{0}}(\boldsymbol{v}, \varrho) \partial_{0} \boldsymbol{\psi}+\boldsymbol{A}_{[\boldsymbol{}}^{\underline{r}}(\boldsymbol{v}, \varrho) c_{\underline{r}} \boldsymbol{\psi}=\boldsymbol{B}_{[\psi]}(\boldsymbol{v}) \boldsymbol{\psi}+\boldsymbol{M}_{[\boldsymbol{}}(\boldsymbol{v}, \boldsymbol{\phi}, \varrho) .
\end{aligned}
$$

In equations (47a)-(47d), E denotes the $5 \times 5$ unit matrix, while $\boldsymbol{A}_{[\boldsymbol{\phi}]}^{\underline{s}}, \boldsymbol{A}_{[\boldsymbol{\varrho}]}^{\frac{s}{4}}, \boldsymbol{A}_{[\boldsymbol{\psi}]}^{\frac{s}{4}}$ denote smooth symmetric matrix-valued functions of their respective arguments. In particular, $\boldsymbol{A}_{[\boldsymbol{\phi}]}^{\frac{0}{}}(\mathbf{0})=\mathbf{0}$ and $\boldsymbol{A}_{[\varrho]}^{\frac{0}{\varrho}}, \boldsymbol{A}_{[\boldsymbol{\psi}]}^{\frac{0}{\boldsymbol{w}}}$ are positive definite if $\rho>0$. In addition, $\boldsymbol{B}_{[\boldsymbol{v}]}, \boldsymbol{B}_{[\boldsymbol{\phi}]}, \boldsymbol{B}_{[\varrho]}$ and $\boldsymbol{B}_{[\boldsymbol{\psi}]}$ are smooth matrixvalued functions of $\boldsymbol{v}$. Finally, $\boldsymbol{M}_{[\boldsymbol{v}]}, \boldsymbol{M}_{[\phi]}$ and $\boldsymbol{M}_{[\boldsymbol{\psi}}$ are non-linear vector-valued functions of their respective arguments. These functions are smooth if $u^{0} \neq 0$.

For convenience of the discussion, in the sequel, we define

$$
\boldsymbol{w} \equiv(\operatorname{Re}(\boldsymbol{v}), \operatorname{Im}(\boldsymbol{v}), \operatorname{Re}(\boldsymbol{\phi}), \operatorname{Im}(\boldsymbol{\phi}), \operatorname{Re}(\varrho), \operatorname{Im}(\varrho), \operatorname{Re}(\boldsymbol{\psi}), \operatorname{Im}(\boldsymbol{\psi}))
$$

Remark 7. The deduction of the evolution equations (47a)-(47d) assumes the choice of gauge source functions given in (46).

\subsection{Propagation of the constraints}

An analysis of the so-called subsidiary equations describing the propagation of the zero quantities

$$
\Sigma_{i j}^{l}, \quad \Xi_{l i j}^{k}, \quad \Delta_{l i j}, \quad \Lambda_{l i j}, \quad \delta_{k}, \quad \delta_{i j}, \quad \zeta_{k}, \quad \zeta
$$

in terms of which the geometric part of the conformal field equations - see equations (20a)(20b) - is expressed has been given in [10. This lengthy analysis is succinctly summarised in the following lemma:

Lemma 5. If the unphysical energy-momentum tensor, $T_{i j}$, satisfies

$$
\nabla^{i} T_{i j}=0
$$

and the expressions

$$
\nabla^{i} T_{i j k}, \quad\left(\frac{1}{2} \Theta^{3} T_{i}^{m} d_{m j k l}+\nabla_{i} \Theta T_{k l j}+\Theta \nabla_{i} T_{k l j}\right) \epsilon_{n}^{i k l}-d^{m} \epsilon^{k l}{ }_{m j} T_{k l n},
$$

can be rewritten in terms of matter zero quantities, then the geometric zero quantities in (48) satisfy a subsidiary system which is symmetric hyperbolic and homogeneous in the zero quantities.

A lengthy computation assuming the form for the energy-momentum tensor given by equation (23) and taking into account the expression (30) for the rescaled Cotton-York tensor, shows that the expressions (49) in Lemma 5] can be rewritten as a homogeneous expressions of the matter zero quantities $q_{k}, y_{i j}, Z_{k j}$ and $Q_{k j}$ defined by equations (31), (34) and (38), respectively. The analogue of Lemma 5 for the matter zero quantities is given by Lemmas 2 and 4 .

The purpose of the analysis of the propagation of the constraints is to establish the following reduction theorem which follows directly from the symmetric hyperbolicity of the subsidiary systems and their homogeneity with respect to the zero quantities. 
Theorem 1. A smooth solution $\boldsymbol{w}$ of the propagation equations 47a - 47b which satisfies the constraint equations on a spacelike hypersurface $\mathcal{S}$ defines in the domain of dependence of $\mathcal{S}$ a solution to the conformal Einstein field equations with matter model given by a traceless perfect fluid.

\section{The traceless perfect fluid FLRW cosmology as a solution to the conformal Einstein field equations}

The purpose of the present section is to cast the traceless perfect fluid FLRW cosmology with $\lambda<0$ in a form in which its character as a solution to the conformal Einstein field equations with matter becomes manifest.

\subsection{The FLRW cosmology on the Einstein cylinder}

One of the most important properties characterising FLRW cosmologies is their conformal flatness. This shows that as in the case of the Minkowski, de Sitter and anti-de Sitter spacetimes, these solutions admit a conformal representation in which the unphysical spacetime $\left(\mathcal{M}, g_{\mu \nu}\right)$ is given by the so-called Einstein cylinder (or Einstein cosmos).

The Einstein cosmos is given by the manifold $\mathcal{M}_{\mathscr{E}}=\mathbb{R} \times \mathbb{S}^{3}$ with a metric given by the line element

$$
g_{\mathscr{E}}=\mathrm{d} \tau^{2}-\mathrm{d} \sigma^{2}
$$

where, again, $d \sigma^{2}$ is the standard line element of $\mathbb{S}^{3}$. The manifold $\mathbb{S}^{3}$ will be coordinatised in the way indicated in Subsection 2.2. A $g$-orthonormal frame $\stackrel{\circ}{e}_{k}$ can be defined on $\mathcal{M}_{\mathscr{E}}$ by completing the frame $\left\{c_{1}, c_{2}, c_{3}\right\}$ discussed also in Subsection 2.2 with the vector $\stackrel{\circ}{e}_{0}=\partial_{\tau}$. Setting, for convenience, $c_{0} \equiv \partial_{\tau}$, one can write $\stackrel{\circ}{e}_{k}=\stackrel{\circ}{e}_{k} \underline{\underline{s}} c_{\underline{s}}$ with $\stackrel{\circ}{e}_{k} \underline{s}=\delta_{k} \underline{s}$ - the components of $\stackrel{\circ}{k}_{k}$ with respect to the basis $c_{\underline{s}}$.

In order to relate the FLRW line element (6) with that of the Einstein cosmos, equation (50), one introduces the change of coordinate

$$
\tau=\int_{t_{0}}^{t} \frac{\mathrm{d} s}{a(s)} .
$$

This naturally leads to the following choice of conformal factor:

$$
\stackrel{\Theta}{ }(\tau) \equiv 1 / a(\tau) \equiv 1 / a(t(\tau)),
$$

so that $g_{\mathscr{E}}=\stackrel{\circ}{\Theta}^{2} \tilde{g}_{\mathscr{F}}$, where $\tilde{g}_{\mathscr{F}}$ is given by equation (6). For the class of FLRW cosmologies

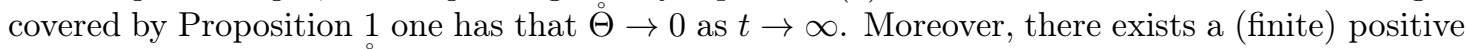
constant $\tau_{\infty}$ such that $\stackrel{\circ}{\Theta}\left(\tau_{\infty}\right)=0$. Notice also that $\tau=0$ for $t=t_{0}$.

A direct computation using the line element (50), the frame $\stackrel{\circ}{k}_{k}$ and the conformal factor (51) gives the following expressions for the unknowns of the conformal field equations:

$$
\begin{aligned}
& \stackrel{\circ}{e}_{k} \underline{s}=\delta_{k} \underline{\underline{s}}, \quad \stackrel{\circ}{\Gamma}_{i k}^{j}=\epsilon_{0 i l k} \eta^{j l}, \quad \stackrel{\circ}{P}_{i j}=\delta_{i}{ }^{0} \delta_{j}{ }^{0}-\frac{1}{2} \eta_{i j}, \quad \stackrel{\circ}{i j k l}_{i j k}=0, \\
& \stackrel{\circ}{\Theta}=a^{-1}, \quad \stackrel{\circ}{d}_{k}=-a^{-2} a^{\prime} \delta_{k}{ }^{0}, \quad \stackrel{\circ}{s}=\frac{1}{2} a^{-3} a^{2}-\frac{1}{4} a^{-2} a^{\prime \prime}-\frac{1}{4} a^{-1}, \\
& \stackrel{\circ}{\rho}=\tilde{\rho}_{0} a_{0}^{4}, \quad \stackrel{\circ}{u}_{i}=\delta_{i}{ }^{0}, \quad \stackrel{\circ}{\rho}_{k}=0, \quad \stackrel{\circ}{u}_{i j}=0 .
\end{aligned}
$$

where ' denotes differentiation with respect to $\tau$, and $a_{0}$ and $\tilde{\rho}_{0}$ are the values of the scale factor and the physical pressure at the initial time $\tau=0$. Notice that the unphysical density for this model is constant. Spinorial versions of the above expressions can be readily obtained by contraction with the constant spacetime Infeld-van der Waerden symbols $\sigma_{A A^{\prime}}$, or their space spinor version $\sigma^{a} A B$. The explicit expressions will not be required in our subsequent analysis. Following the notation of Lemma 7, we collect the independent spinorial components of the fields in $(52 \mathrm{a})-(52 \mathrm{c})$ in a vectorial unknown which we denote by $\stackrel{\boldsymbol{w}}{\text {. }}$ 
A direct computation using the expressions (52a)-(52C) shows that, for this solution, the gauge source functions $F^{\mu}, F_{(A B)}$ and $F_{A A^{\prime}}$ as defined by (45a)-(45c) are given by

$$
F^{\mu}=0, \quad F_{(A B)}=0, \quad F_{A A^{\prime}}=0 .
$$

This computation justifies the choice of gauge source functions made in (46).

Recalling that $\mathrm{d} / \mathrm{d} \tau=a \mathrm{~d} / \mathrm{d} t$, and using the limits given in Proposition 1 it follows that

$$
\stackrel{\circ}{d}_{k} \rightarrow-\sqrt{-\frac{1}{3} \lambda}, \quad \stackrel{\circ}{s} \rightarrow 0 \quad \text { as } \tau \rightarrow \tau_{\infty} .
$$

Accordingly, the expressions given in (52a), (52b) and (52c) define a smooth solution to the conformal Einstein field equations (20a)-(20b) for $\tau \in\left[0, \tau_{\infty}\right]$. In fact, this solution extends, at least locally, beyond $\tau=\tau_{\infty}$. This can be easily seen to be the case by using the values of the solution (52a)-(52c) as the initial value for a Cauchy problem on the slice $\tau=\tau_{\infty}$. The symmetric hyperbolicity of the evolution equations implies that the solution to this initial value problem exists for $\tau \in\left[\tau_{\infty}, \tau_{\infty}+\delta\right.$ ) for some $\delta>0$. From the expression for $\stackrel{d}{k}_{k}$ in (52b) and Proposition 1 it follows that $\AA_{0}<0$ at $\tau_{\infty}$. Thus, by continuity, $\delta$ can be chosen such that $\Theta<0$ on $\left(\tau_{\infty}, \tau_{\infty}+\delta\right)$. In summary we have:

Lemma 6. There exists $\delta>0$ such that the expressions (52a)-(52c) give rise to a solution to the evolution equations implied by the conformal Einstein field equations (20a)-(20b) on $\left[0, \tau_{\infty}+\delta\right.$ ). Furthermore $\stackrel{\Theta}{\Theta} 0$ in $\left(\tau_{\infty}, \tau_{\infty}+\delta\right)$.

\section{Initial data for a FLRW cosmology}

The expressions in (52a)-(52c) naturally induce an initial data set for the conformal Einstein field equations which we denote by $\boldsymbol{\boldsymbol { w }}_{0}$. Notice that there is no need for performing a pull-back in this construction as the fields in (52a)-(52c) are all scalars.

\subsection{Structure of the conformal boundary}

The structure of the conformal boundary for the solution to the conformal Einstein field equations described by (52a)-(52C) follows directly by inspection.

By construction at $\tau=\tau_{\infty}$ one has that $\stackrel{\circ}{\Theta}=0$. From the limits (53) - see also equation (19h) - one has that:

$$
\stackrel{\circ}{d}_{k} \stackrel{\circ}{d}^{k}=\nabla_{k} \stackrel{\circ}{\Theta} \nabla^{k} \stackrel{\ominus}{\Theta}=-\frac{1}{3} \lambda>0, \quad \text { at } \quad \tau=\tau_{\infty},
$$

so that the future conformal boundary $\mathscr{I}^{+} \equiv\left\{p \in \mathcal{M}_{\mathscr{E}} \mid \stackrel{\Theta}{\Theta}=0\right\}$ is spacelike.

\section{Existence and stability results}

The purpose of the present section is to provide our main results. These concern the global existence of solutions to conformal Einstein field equations with matter source given by a perfect fluid in the case $\lambda<0, \gamma=\frac{4}{3}$ which can be regarded as non-linear perturbations of the reference FLRW solution described by Proposition 1. We also provide results concerning the structure of the conformal boundary for these solutions. Altogether these results show the non-linear stability towards the future of the reference FLRW cosmological model.

\subsection{An Ansatz for the solution}

We will consider solutions to the evolution equations (47a)-(47d) of the form $\boldsymbol{w}=\stackrel{\boldsymbol{w}}{ }+\breve{\boldsymbol{w}}$, where

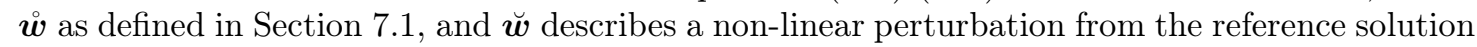

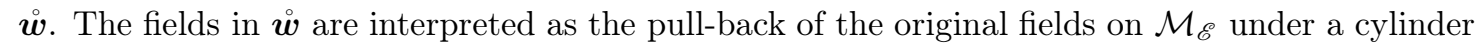
map. In what follows let $\boldsymbol{w}_{0}=\stackrel{\boldsymbol{w}}{0}_{0}+\breve{\boldsymbol{w}}_{0}$ be an initial data set for the system of evolution equations (47a)-(47d) prescribed on the initial manifold $\mathcal{S}$-as discussed in Subsection 8.2 It 
will be assumed that $\boldsymbol{w}_{0}$ satisfies the conformal constraint equations. The vector $\dot{\boldsymbol{w}}_{0}$ is to be interpreted as the pull-back of the smooth map relating $\mathbb{S}^{3}$ and the initial manifold $\mathcal{S}$.

A direct inspection gives rise to the following result:

Lemma 7. For $\breve{\boldsymbol{w}}$ sufficiently close to $\mathbf{0}$ and as long as $\rho>0$ and $u^{0} \neq 0$, the equations (47a)(47d) imply a symmetric hyperbolic evolution system

$$
\boldsymbol{A}^{0}(\stackrel{\circ}{\boldsymbol{w}}+\breve{\boldsymbol{w}}) \cdot \partial_{\tau} \breve{\boldsymbol{w}}+\sum_{\underline{r}=1}^{3} \boldsymbol{A}^{\underline{r}}(\stackrel{\circ}{\boldsymbol{w}}+\breve{\boldsymbol{w}}) \cdot c_{\underline{r}}(\breve{\boldsymbol{w}})+\boldsymbol{B}\left(\tau, \boldsymbol{x}, \stackrel{\circ}{\boldsymbol{w}}, c_{\underline{s}} \stackrel{\circ}{\boldsymbol{w}}, \breve{\boldsymbol{w}}\right) \cdot \breve{\boldsymbol{w}}=0
$$

for the independent components of $\boldsymbol{w}$. The matrix valued functions $\boldsymbol{A}^{\mathrm{s}}, \boldsymbol{B}$ are smooth functions of their arguments. Furthermore, the entries of the matrix $\boldsymbol{A}^{0}(\boldsymbol{w})$ are bounded from below by $1 / \sqrt{2}$. Finally, $\breve{\boldsymbol{w}}=\mathbf{0}$ is a solution of equation (54).

\subsection{Constructing initial data for the conformal evolution equations}

In the sequel, it will be assumed that one has a solution $\left(\mathcal{S}, \tilde{h}_{\alpha \beta}, \tilde{K}_{\alpha \beta}, \tilde{\rho}, \tilde{u}^{\alpha}\right)$ to the (physical) $\lambda<0$ Einstein-perfect fluid constraint equations

$$
\begin{aligned}
& \tilde{r}+\tilde{K}^{2}-\tilde{K}_{\alpha \beta} \tilde{K}^{\alpha \beta}=2(\lambda-\tilde{\mu}), \\
& \tilde{D}^{\alpha} \tilde{K}_{\alpha \beta}-\tilde{D}_{\beta} \tilde{K}=\tilde{j}_{\beta},
\end{aligned}
$$

with $\mathcal{S}$ having the topology of $\mathbb{S}^{3}$ and the perfect fluid satisfying a barotropic equation of state with $\gamma=\frac{4}{3}$. In equations (55a)-(55b) $\tilde{D}_{\beta}$ and $\tilde{r}$ denote the Levi-Civita covariant derivative and the Ricci scalar of the intrinsic 3-metric $\tilde{h}_{\alpha \beta}$ of $\mathcal{S} . \tilde{K}_{\alpha \beta}$ is a symmetric 3-dimensional tensor corresponding to the extrinsic curvature of $\mathcal{S}$ with respect to the $\tilde{g}$-unit normal $\tilde{n}_{\mu}$. Furthermore, $\tilde{\mu} \equiv \tilde{n}^{\mu} \tilde{n}^{\nu} \tilde{T}_{\mu \nu}$, while $\tilde{j}_{\beta}$ corresponds to the pull-back to $\mathcal{S}$ of $\tilde{j}_{\lambda} \equiv \tilde{n}^{\mu} \tilde{h}_{\lambda}{ }^{\nu} \tilde{T}_{\mu \nu}$. For a perfect fluid with a tracefree energy-momentum tensor, a direct computation gives that

$$
\tilde{\mu}=\frac{1}{3} \tilde{\rho}\left(4 \tilde{u}_{\|}-1\right), \quad \tilde{j}_{\beta}=\frac{4}{3} \tilde{\rho} \tilde{u}_{\|} \tilde{u}_{\beta},
$$

where $\tilde{u}_{\|} \equiv \tilde{u}^{\mu} \tilde{n}_{\mu}$ and $\tilde{u}_{\beta}$ corresponds to the pull-back of $\tilde{h}_{\mu}{ }^{\nu} \tilde{u}_{\nu}$ to $\mathcal{S}$. In particular, if on $\mathcal{S}$ one has that $\tilde{n}_{\mu}$ and $\tilde{u}^{\nu}$ are aligned - as in the case of the FLRW cosmologies - then $\tilde{\mu}=\tilde{\rho}$ and $\tilde{j}_{\beta}=0$. In general, however, we will consider perfect fluid configurations for which $\tilde{n}_{\mu}$ and $\tilde{u}^{\nu}$ are not aligned.

Using a generalisation of the procedure for vacuum spacetimes described in, say, [6] one can construct a solution to the conformal constraint equations implied on $\mathcal{S}$ by equations (20a)-(20b). Following the notation introduced in Section 6.2 we denote the independent components of such a solution by $\boldsymbol{w}_{0}$.

\subsection{The main result}

In what follows, given $m \in \mathbb{N}$, let $\|\cdot\|_{m}$ denote the Sobolev-like norm on the space $C^{\infty}\left(\mathbb{S}^{3}, \mathbb{R}^{N}\right)$ of smooth $\mathbb{R}^{N}$ valued functions on $\mathbb{S}^{3}$ for some non-negative integer $N$ - see e.g. [8, 18] for precise definitions. Furthermore, let $H^{m}\left(\mathbb{S}^{3}, \mathbb{R}^{N}\right)$ be the Hilbert space obtained as the completion of the space $C^{\infty}\left(\mathbb{S}^{3}, \mathbb{R}^{N}\right)$ in the norm $\|\cdot\|_{m}$. Using the cylinder map between $\mathcal{M}_{\mathscr{E}}$ and $\mathcal{M}$, one can apply the norm $\|\cdot\|_{m}$ to evaluate the norm of functions on the unphysical initial hypersurface $\mathcal{S}$. Furthermore, the vector $\breve{\boldsymbol{w}}=\breve{\boldsymbol{w}}(\tau, \boldsymbol{x})$ can be regarded as a function of $\tau$ which takes values in $H^{m}\left(\mathbb{S}^{3}, \mathbb{R}^{N}\right)$.

Our main result is the following theorem:

Theorem 2. Suppose $m \geq 4$. Let $\mathcal{S}$ denote a 3-dimensional manifold diffeomorphic to $\mathbb{S}^{3}$, and let $\boldsymbol{w}_{0}=\stackrel{\boldsymbol{w}}{0}_{0}+\breve{\boldsymbol{w}}_{0}$ be initial data for the conformal evolution equations (47a)-(47d) constructed from some physical initial data set, $\left(\mathcal{S}, \tilde{h}_{\alpha \beta}, \tilde{K}_{\alpha \beta}, \tilde{\rho}, \tilde{u}^{\alpha}\right)$, for the Einstein field equations with $\lambda<0$ and matter source given by a traceless perfect fluid $(\gamma=4 / 3)$. There exists $\varepsilon>0$ such that if 
$\left\|\breve{\boldsymbol{w}}_{0}\right\|_{m}<\varepsilon$ then the initial data set $\boldsymbol{w}_{0}$ determines a unique solution, $\boldsymbol{w}$, to the evolution equations (47a) - (47dd) which exists on $\left[0, \tau_{*}\right]$ with $\tau_{*}>\tau_{\infty}$. The solution $\boldsymbol{w}$ is of class $C^{m-2}\left(\left[0, \tau_{*}\right] \times \mathbb{S}^{3}\right)$ is such that:

(i) it determines, in turn, a $C^{m-2}$ solution to the $\lambda<0$ conformal Einstein field equations, equations (20a)-(20b) and (25)-(34), with matter given by a traceless fluid on $\left[0, \tau_{*}\right] \times \mathbb{S}^{3}$;

(ii) there exists a function $\tau_{+}=\tau_{+}(\boldsymbol{x}), \boldsymbol{x} \in \mathbb{S}^{3}$, such that $0<\tau_{+}(\boldsymbol{x})<\tau_{*}$ and

$$
\begin{aligned}
& \Theta>0, \quad \text { on } \quad \tilde{\mathcal{M}} \equiv\left\{(\tau, \boldsymbol{x}) \in \mathbb{R} \times \mathbb{S}^{3} \mid 0 \leq \tau<\tau_{+}(\boldsymbol{x})\right\}, \\
& \Theta=0, \quad d_{k} d^{k}=-\frac{1}{3} \lambda<0 \quad \text { on } \quad \mathscr{I}^{+} \equiv\left\{\left(\tau_{+}(\boldsymbol{x}), \boldsymbol{x}\right) \in \mathbb{R} \times \mathbb{S}^{3} \mid \boldsymbol{x} \in \mathbb{S}^{3}\right\} .
\end{aligned}
$$

(iii) one obtains a $C^{m-2}$ solution $\left(\tilde{\mathcal{M}}, \tilde{g}_{\mu \nu}, \tilde{\rho}, \tilde{u}^{\mu}\right)$, to the $\lambda<0$ Einstein-perfect fluid field equations with $\gamma=4 / 3$ which is future geodesically complete for which $\mathscr{I}^{+}$as defined above represents conformal future infinity;

(iv) given a sequence of initial data $\boldsymbol{w}_{0}^{(n)}$ such that $\left\|\breve{\boldsymbol{w}}_{0}^{(n)}\right\|_{m}<\varepsilon$ and $\left\|\breve{\boldsymbol{w}}_{0}^{(n)}\right\|_{m} \rightarrow 0$ as $n \rightarrow$ $\infty$, then for the corresponding solutions $\breve{\boldsymbol{w}}^{(n)}$ (with minimum existence time $\tau_{*}$ ) one has $\left\|\breve{\boldsymbol{w}}^{(n)}\right\|_{m} \rightarrow 0$ uniformly in $\tau \in\left[0, \tau_{*}\right]$.

Remark 8. The above theorem, and in particular part (iv), amounts to a non-linear stability result for the $\lambda<0, \gamma=\frac{4}{3}$ FLRW cosmological models, in the sense that sufficiently small perturbations of data for the FLRW solution give rise to (future) global solutions to the Einstein field equations with the same asymptotic structure as the reference solution.

Remark 9. Note that no consideration of the vorticity of the radiation fluid was required for the derivation. The vorticity of the fluid can be calculated from the components of $u_{i j}$.

Proof. Existence, uniqueness and the smoothness of the solutions to equations (47a)-(47d) follow from the properties of the equation (54) provided in Lemma 7 and an extension of the general existence and stability Theorem by Kato [16] provided in [8] - see also [18. In particular, if $\varepsilon$ is sufficiently small, one obtains a common existence time $\tau_{*}>\tau_{\infty}$ for all initial data with $\left\|\breve{\boldsymbol{w}}_{0}\right\|_{m}<\varepsilon$. Part (iv) follows from the same result.

Now, the Reduction Theorem, Theorem 1 ensures that if the conformal constraint equations are satisfied on $\mathcal{S}$, one obtains a $C^{m-2}$ solution on $\left[0, \tau_{*}\right] \times \mathbb{S}^{3}$ to the $\lambda<0$ conformal Einstein perfect fluid equations with $\gamma=\frac{4}{3}$ - this shows part (i).

In order to show part (ii), one observes that because $\Theta<0$ in $\left(\tau_{\infty}, \tau_{\infty}+\delta\right)$, then if $\varepsilon$ is sufficiently small one has that $\Theta<0$ at, say, $\tau=\tau_{\infty}+\delta / 2$. As $\Theta>0$ at $\tau=0$, then there is a $\tau_{+}$ for which $\Theta=0$. By reducing, if necessary $\varepsilon$ one has that such $\tau$ is unique, and hence, the function $\tau_{+}(\boldsymbol{x})$ is well defined. If follows from (19e) and (19h) that $\Theta=0$ implies $d_{k} d^{k}=\nabla_{k} \Theta \nabla^{k} \Theta=$ $-\frac{1}{3} \lambda>0$. Hence $\Theta=0$, respectively $\tau=\tau_{+}(\boldsymbol{x})$, defines a regular spacelike hypersurface $\mathscr{I}^{+}$.

For part (iii) one notices that a solution of the vacuum conformal Einstein field equations implies a solution to the vacuum Einstein field equations - see e.g. 6. 10. If $\omega^{k}$ denotes the dual cobasis of the frame $e_{i},\left\langle\omega^{k}, e_{i}\right\rangle=\delta^{k}{ }_{i}$, then the unphysical metric is given by $g=\eta_{i j} \omega^{i} \otimes \omega^{j}$. If $\varepsilon$ is sufficiently small, one has that $\operatorname{det}(g) \neq 0$ on $\left[0, \tau_{*}\right]$ as $\operatorname{det}(\stackrel{g}{g}) \neq 0$. Thus $\tilde{g}=\Theta^{-2} g$ is well defined on $\tilde{\mathcal{M}}$. The matter fields $\tilde{\rho}$ and $\tilde{u}^{\mu}$ are defined via the formulae in (21). An adaptation of this argument to our setting gives the desired result. Geodesic completeness follows from an analysis of the geodesic equations and standard perturbative arguments for ordinary equations given that the background solution is future geodesically complete. 


\section{Acknowledgements}

Part of this research was carried out at the Erwin Schrödinger International Institute for Mathematical Physics of the University of Vienna, Austria, during the course of the programme "Dynamics of General Relativity: Numerical and Analytical Approaches" (July-September, 2011) and the workshop "Cartan connections, geometry of homogeneous spaces, and dynamics" (July 2011). The authors thank the organisers for the invitation to attend these programmes and the institute for its hospitality. We have profited from interesting discussions with Prof. H. Friedrich. C.L. would like to thank Queen Mary University of London for a Visiting Fellowship.

\section{References}

[1] K. Anguige \& K. P. Tod, Isotropic Cosmological Singularities I. Polytropic Perfect Fluid Spacetimes, Ann. Phys. 276, 257 (1999).

[2] J. Bičák, M. Scholtz, \& K. P. Tod, On asymptotically flat solutions of Einstein's equations periodic in time: II. Spacetimes with scalar-field sources, Class. Quantum Grav. 27, 175011 (2010).

[3] Y. Choquet-Bruhat, General Relativity and the Einstein equations, Oxford University Press, 2008.

[4] H. Friedrich, The asymptotic characteristic initial value problem for Einstein's vacuum field equations as an initial value problem for a first-order quasilinear symmetric hyperbolic system, Proc. Roy. Soc. Lond. A 378, 401 (1981).

[5] H. Friedrich, On the regular and the asymptotic characteristic initial value problem for Einstein's vacuum field equations, Proc. Roy. Soc. Lond. A 375, 169 (1981).

[6] H. Friedrich, Cauchy problems for the conformal vacuum field equations in General Relativity, Comm. Math. Phys. 91, 445 (1983).

[7] H. Friedrich, On the hyperbolicity of Einstein's and other gauge field equations, Comm. Math. Phys. 100, 525 (1985).

[8] H. Friedrich, On purely radiative space-times, Comm. Math. Phys. 103, 35 (1986).

[9] H. Friedrich, On the existence of n-geodesically complete or future complete solutions of Einstein's field equations with smooth asymptotic structure, Comm. Math. Phys. 107, 587 (1986).

[10] H. Friedrich, On the global existence and the asymptotic behaviour of solutions to the Einstein-Maxwell-Yang-Mills equations, J. Diff. Geom. 34, 275 (1991).

[11] H. Friedrich, Einstein equations and conformal structure: existence of anti-de Sitter-type space-times, J. Geom. Phys. 17, 125 (1995).

[12] H. Friedrich, Evolution equations for gravitating ideal fluid bodies in general relativity, Phys. Rev. D 57, 2317 (1998).

[13] H. Friedrich, Conformal Einstein evolution, in The conformal structure of spacetime: Geometry, Analysis, Numerics, edited by J. Frauendiener \& H. Friedrich, Lecture Notes in Physics, page 1, Springer, 2002.

[14] J. B. Griffiths \& J. Podolský, Exact space-times in Einstein's General Relativity, Cambridge University Press, 2009.

[15] P. Hübner, General relativistic scalar-field models and asymptotic flatness, Class. Quantum Grav. 12, 791 (1995). 
[16] T. Kato, The Cauchy problem for quasi-linear symmetric hyperbolic systems, Arch. Ration. Mech. Anal. 58, 181 (1975).

[17] H.-O. Kreiss \& J. Lorenz, Stability for time-dependent differential equations, Acta Numerica $7(203)$ (1998).

[18] C. Lübbe \& J. A. Valiente Kroon, On de Sitter-like and Minkowski-like spacetimes, Class. Quantum Grav. 26, 145012 (2009).

[19] C. Lübbe \& J. A. Valiente Kroon, A stability result for purely radiative spacetimes, J. Hyp. Diff. Eqns. 7, 545 (2010).

[20] C. Lübbe \& J. A. Valiente Kroon, The extended Conformal Einstein field equations with matter: the Einstein-Maxwell system, 2011.

[21] R. Penrose \& W. Rindler, Spinors and space-time. Volume 1. Two-spinor calculus and relativistic fields, Cambridge University Press, 1984.

[22] A. D. Rendall, The initial value problem for a class of general relativistic fluid bodies, J. Math. Phys. 33, 1047 (1992).

[23] O. Reula, Exponential decay for small nonlinear perturbations of expanding flat homogeneous cosmologies, Phys. Rev. D 60, 083507 (1999).

[24] I. Rodnianski \& J. Speck, The Stability of the Irrotational Euler-Einstein System with a Positive Cosmological Constant, In arXiv:0911.5501, 2009.

[25] P. Sommers, Space spinors, J. Math. Phys. 21, 2567 (1980).

[26] J. Speck, The Nonlinear Future-Stability of the FLRW Family of Solutions to the EulerEinstein System with a Positive Cosmological Constant, In arXiv:1102.1501, 2011. 